\title{
The Expression of Dielectric Losses in Cables
}

\author{
BY WM. A. DEL MAR \\ Chief Engineer, Habirshaw Electric Cable Co.
}

$D^{1}$ IELECTRIC losses in cables depend upon the characteristics of the current, the dimensions of the cable and the specific qualities of the insulation. In order to compare the specific qualities of different insulations with respect to dielectric losses, it is désirable to find a method of expressing these specific qualities independently of current characteristics and cable dimensions. This will permit different insulations to be compared even when tests are made at different voltages, or on cables of different sizes. A method of attaining this result is here suggested.

The method consists in expressing the specific quality of the insulation with respect to dielectric losses, in terms of the product of its power-factor and specific inductive capacity. This may be termed the coefficient of dielectric loss and is hereinafter designated by the letter $K_{\mathrm{L}}$.

If the specific inductive capacity or specific capacitance may be considered to have a maximum value of 3.5 for impregnated paper insulation, the coefficient of dielectric loss must be between zero and 3.5, the value zero corresponding to zero power factor or perfect insulation, and the value 3.5, to unity power factor or the worst insulation. Hence the lower the value of $K_{\mathrm{L}}$, the better the insulation from the point of view of dielectric losses. The following values are characteristic of different makes of insulation.

\begin{tabular}{|c|c|c|c|c|c|c|}
\hline \multirow{2}{*}{$\begin{array}{l}\text { Tempera- } \\
\text { ture deg. } \\
\text { cent. }\end{array}$} & \multicolumn{6}{|c|}{ Cable No. } \\
\hline & I & II & III & IV & V & $V I$ \\
\hline 50 & 0.093 & 0.12 & 0.30 & 0.22 & 0.31 & 0.31 \\
\hline 70 & 0.32 & 0.43 & 0.55 & 0.65 & 0.78 & 0.90 \\
\hline 90 & 0.67 & 0.89 & 1.57 & 1.59 & 1.68 & 1.80 \\
\hline 110 & 1.13 & 1.51 & 2.98 & 2.93 & 2.96 & 2.93 \\
\hline
\end{tabular}

It is proposed for specification purposes, that the Institute standard maximum temperature be adopted, and that, unless otherwise stated, $K_{\mathrm{L}}$ shall refer to that temperature, which is given as 85 deg. cent. for paper in the 1918 Standardization Rules. ${ }^{1}$

The coefficient of dielectric loss may be derived as follows from single-phase measurements of dielectric loss on a triplex cable.

Fig. 1 shows a diagrammatic representation of the capacities in a triplex cable.

Let $I=$ charging current in line, per mile of cable $I_{c}=$ charging current per mile from one conductor to each other conductor

1. Rule 677 of the 1918 Standardization Rules says that the maximum safe temperature, in degrees cent., at the surface of the conductor shall be $85-E$, where $E$ represents the r. m. s. operating e. m. f. in kilovolts between conductors. It is proposed to make $85 \mathrm{deg}$. cent. the temperature of reference whatever the voltage of the cable.
$I_{s}=$ charging current per mile from each conductor to sheath

$E=$ kilovolts between conductors

$C_{c}=$ microfarads per mile one conductor to another

$C_{s}=$ microfarads per mile one conductor to sheath

Then $I=\sqrt{3} I_{c}+I_{0}$

$I_{c}=2 \pi f E C_{c}$ in milli-amperes

$I_{s}=2 \pi f E / 3 \quad C_{s}$ in milli-amperes

$I=2 \pi \sqrt{3} f E\left(C_{c}+C_{s} / 3\right)$

Let $\quad w=$ dielectric loss, watts per mile of cable

Then

$\theta=$ power factor angle

$w=\sqrt{3} E I \cos \theta$
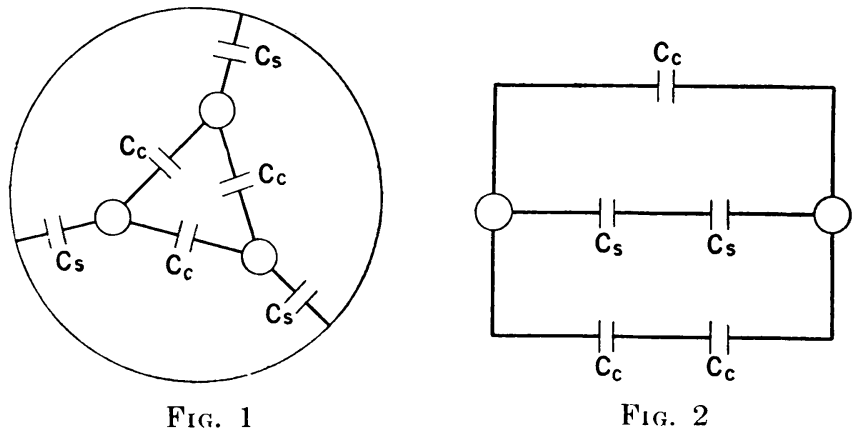

Combining equations 1 and 2

$$
w=2 \pi f E^{2} \cos \theta\left(3 C_{c}+C_{s}\right)
$$

Now $C_{c}$ and $C_{s}$ are purely mathematical conceptions not susceptible of direct measurement. They are the capacities of imaginary condensers. The real capacities differ due to being composed of groups of these imaginary capacities in series or parallel. We must therefore reduce these imaginary capacities to real capacities.

Let $C_{1}=$ actual capacity (as measured) between any two conductors, the third being insulated.

Then $C_{1}$ is composed of the combination of $C$. and $C_{c}$ shown in Fig. 2.

$$
\begin{aligned}
C_{1} & =11 / 2 C_{c}+1 / 2 C_{s} \\
& =1 / 2\left(3 C_{c}+C_{s}\right)
\end{aligned}
$$

or

$$
\left(3 C_{c}+C_{s}\right)=2 C_{1}
$$

Combining equations (3) and (4)

$$
w=4 \pi f E^{2} \cos \theta . C_{1}
$$

Let $k=$ specific capacitance

Then

$$
\text { and } C=C_{1} / k
$$

Let $K_{\mathrm{L}}=k \cos \theta$

$$
w=4 \pi f E^{2} \cos \theta . k . C
$$

Then,

$$
w=4 \pi f E^{2} K_{\mathrm{L}} C
$$


Let $W=$ watts lost per foot of cable

Then

$$
\begin{aligned}
W & =\frac{4 \pi}{5280} f E^{2} K_{\mathrm{L}} C \\
& =0.00238 f E^{2} K_{\mathrm{L}} C \\
\text { or } K_{\mathrm{L}} & =\frac{420 w}{f E^{2} C}
\end{aligned}
$$

The coefficient $C$, depending only upon the size and shape of conductor and upon the thickness of insulation may be derived either from a general table such as Table II or calculated from a formula such as the one which follows:

$$
C=\frac{0.01945}{A}
$$

$$
\text { Then } \begin{aligned}
i & =\sqrt{3} i_{c}+i \\
i_{c} & =E / R_{c} \\
i_{s} & =\frac{E}{\sqrt{3} R_{s}}
\end{aligned}
$$

Therefore $i=E / \sqrt{3}\left(3 / R_{c}+i / R_{s}\right)$

These resistances are purely imaginary conceptions. Let $R$ be the actual resistance from one conductor to another, the other conductor and sheath not being in circuit. All resistances are expressed in ohm-miles.

Then

$$
\begin{gathered}
R=\frac{2}{\left(3 / R_{c}+1 / R_{s}\right)} \\
\text { or }\left(3 / R_{c}+1 / R_{s}\right)=2 / R \\
\therefore i=2 / \sqrt{3} \times E / R
\end{gathered}
$$

TABLE II

VALUES OF $C$

Actual Capacity of Triplex Cable as measured between any two conductors, the third being insulated (Sp. Cap. = 1). Microfarads per mile.

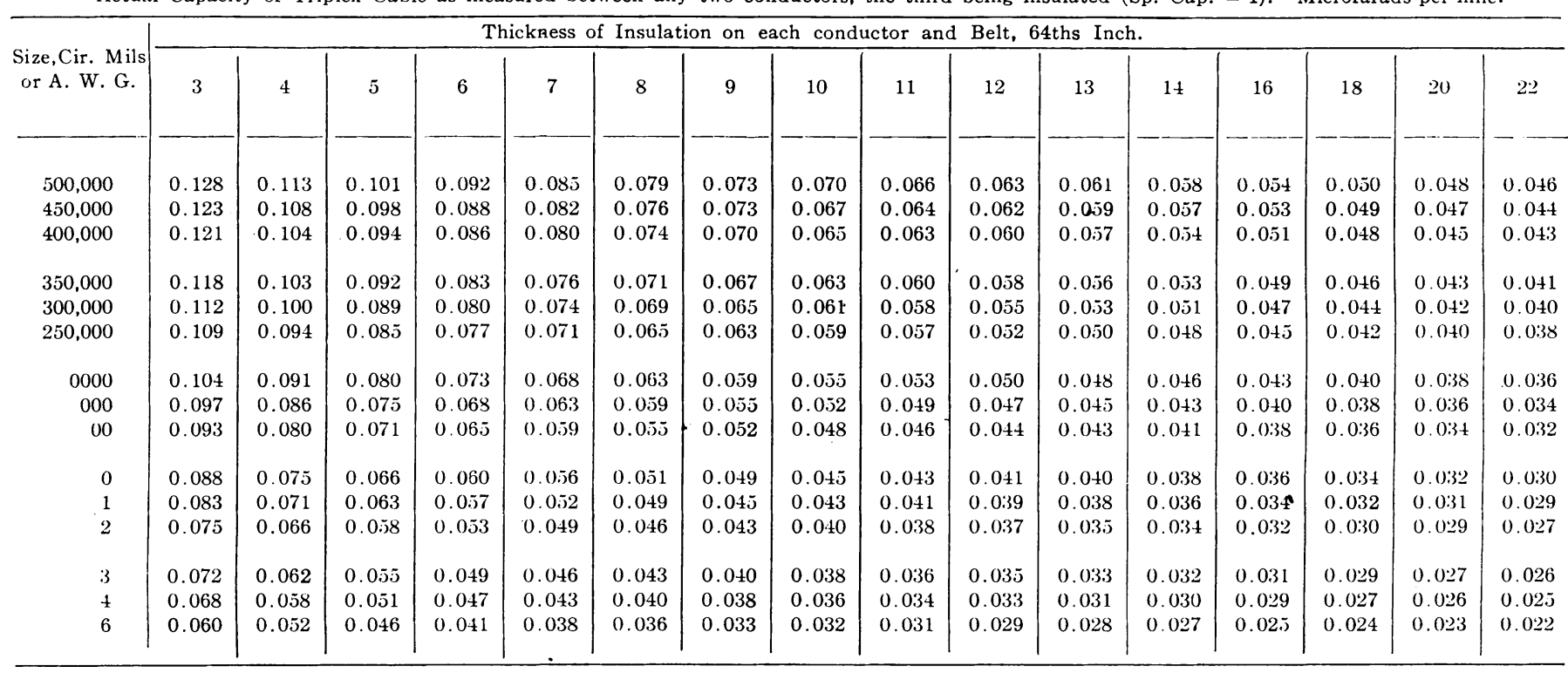

where $A=\log _{10}\left[\frac{d}{a} \frac{b^{2}-d^{2}}{\sqrt{b^{4}+b^{2} d^{2}+d^{4}}}\right]$

in which $a=$ radius of conductor, $\mathrm{cm}$.

$b=$ inside radius of sheath, $\mathrm{cm}$.

$d=$ distance between centers of conductors.

The above calculations are based upon the assumption that the ohmic losses are so low that it is not necessary to take into account the fact that they are independent of the frequency.

This may be easily shown.

Let $i=$ amperes per mile of leakage current, per conductor

$i_{c}=$ amperes per mile of leakage current from one conductor to another

$i_{\text {s }}=$ amperes per mile of leakage current from one conductor to sheath

$R_{c}=$ ohms per mile resistance, one conductor to another

$R_{\mathrm{a}}=$ ohms per mile resistance, one conductor to sheath
Let $W_{0}=$ watts lost in length having resistance $R$

$$
\begin{aligned}
& =\sqrt{3} E \cdot i . \\
& =\frac{2 \sqrt{3}}{\sqrt{3}} \frac{E^{2}}{R} \\
& =2 E^{2} / R
\end{aligned}
$$

If $E=$ kilovolts between conductors

$$
\begin{aligned}
R & =\text { megohm-miles } \\
W & =\text { watts per foot, oh } \\
W & =\frac{2}{5280} \frac{E^{2} \times 10^{6}}{R \times 10^{6}} \\
& =\frac{1}{2640} \frac{E^{2}}{R}
\end{aligned}
$$$$
W=\text { watts per foot, ohmic loss }
$$

Suppose $R=10$ and

$$
E=12
$$

$$
W=\frac{10}{2640}=0.0038 \text { which is negligible in com- }
$$

parison with the loss due to the capacity current. 